Article

\title{
Detection of Gas-Solid Two-Phase Flow Based on CFD and Capacitance Method
}

\author{
Wanting Zhou ${ }^{1,2}$, Yue Jiang ${ }^{3}$, Shi Liu ${ }^{1, *}$, Qing Zhao ${ }^{1}$, Teng Long ${ }^{2}$ and Zhixiong Li $^{4}$ (i) \\ 1 School of Control and Computer Engineering, North China Electric Power University, Beijing 102206, China; \\ wz297@cam.ac.uk (W.Z.); zhaoqing@ncepu.edu.cn (Q.Z.) \\ 2 Department of Engineering, University of Cambridge, Cambridge CB3 0FA, UK; TL322@cam.ac.uk \\ 3 School of Energy, Power and Mechanical Engineering, North China Electric Power University, \\ Beijing 102206, China; jiangyue@ncepu.edu.cn \\ 4 School of Mechanical, Materials, Mechatronic and Biomedical Engineering, University of Wollongong, \\ Wollongong, NSW 2522, Australia; zhixiong.li@ieee.org \\ * Correspondence: liushi@ncepu.edu.cn
}

Received: 15 July 2018; Accepted: 7 August 2018; Published: 14 August 2018

check for updates

\begin{abstract}
Multiphase flow in annular channels is complex, particularly in the region where the flow direction abruptly changes between the inner pipe and the outer pipe, as the cases in horizontal drilling and pneumatic conveying. Simplified models and experience are still the main sources of information. First, to understand the process more deeply, Computational Fluid Dynamics (CFD) package Fluent is used to simulate the gas-solid flow in the horizontal and the inclined section of an annular pipe. Discrete Phase Model (DPM) is adopted to calculate the trajectories of solid particles of different sizes at different air velocities. Also, the Two-Fluid model is used to simulate the sand flow in the inclined section for the case of air flow stoppage, for which an experiment is also conducted to verify the CFD simulation. Simulation results reveal the behaviour of the solid particles showing the dispersed spatial distribution of small particles near the entrance. On the other hand, larger particles manifest a distinct sedimented flow pattern along the bottom of the pipe. The density distribution of the particles over a pipe cross section is demonstrated at a variety of air velocities. The results also show that the large airspeed tends to generate swirls near the outlet of the inner pipe. In addition, Electrical Capacitance Tomography (ECT) technology is used to reconstruct the spatial distribution of particles, and the cross-correlation algorithm to detect velocity. Both the distribution and the velocity measurement by electric sensors agree reasonably well with the CFD predictions. The details revealed by CFD simulation and the mutual-verification between CFD simulation and the ECT method of this study could be valuable for the industry in drilling process control and equipment development.
\end{abstract}

Keywords: Discrete Phase Model (DPM); Two-Fluid model; numerical simulation; annular space; cross-correlation

\section{Introduction}

Gas-solid two-phase flow is common in industrial applications. While the majority of studies are focused on the flows in circular pipes, flow in annular pipes has much to be investigated due to its frequent appearances in the oil industry, such as in horizontal drilling.

Along with experimental studies, numerical simulation is becoming the main tool for gas-solid flows in the oil industry. Many researchers have made many efforts to study the flow via numerical simulations. Gargano [1] studied the various viscous-inviscid interactions that occur during the unsteady separation process. The study showed how the van Dommelen and Shen singularity that occurs in solutions of the Prandtl Boundary-layer Equations evolves in the complex plane before 
leading to a separation singularity in finite time. Obabkop [2] studied unsteady separation induced by a vortex using Navier-Stokes Equation. The numerical solutions of the Navier-Stokes Equations show that the interaction occurs on two distinct streamwise length scales depending upon which of three Reynolds-number Regimes is being considered. Clercx [3] studied the dipolar vortex in two-dimensional turbulent flows. He found the dipolar vortex propagates easily through the domain and hence, is likely to interact with domain boundaries. The collision of such structures with rigid domain boundaries is investigated in standard vortex generation mechanisms.

Concerning the cases of the two-phase flow in drilling pipes in the oil industry, Chen, Zou et al. [4] studied downhole cuttings adhesion of Polycrystalline Diamond Compact (PDC) bit based on Discrete Phase Model (DPM). Huang, Wang et al. [5] studied local erosion and abrasion of cutting-convey pipe elbow in gas drilling. It is found that the abrasion rate of the pipe elbow with the angle from $30^{\circ}$ to $60^{\circ}$ is much higher than the average by numerical simulation. According to the distribution feature of elbow abrasion, the structural modification of pipe elbow is conducted by putting a baffle in the main abrasion position of the wall surface. Zhu et al. [6] analysed the distribution of cuttings on the Down-The-Hole (DTH) drilling bit with the DPM model, and the influence on air flow by the structure of drilling bit was analysed. The improved structure of drilling bit was also presented to clear the rock timely, extend the life of bit and improve the speed of drilling. Zhang et al. [7] optimised cylindrical teeth's topology of DTH bit based on the analyses of gas-solid two-phase flow. There are also plenty of studies about gas-solid flow applied in other areas. Sedrez [8] studied the erosion modeling for gas-solids flow in cyclones via experiments and simulation. The numerical simulation in this study was performed using the Euler-Lagrange approach with the Reynolds Stress Model (RSM) for turbulence in the gas phase, with two-way coupling to take into account the gas-solid interaction, and two erosion models. The experiment's results showed an increase in the erosion with the gas inlet velocity in the cyclone, notably at velocities of 30 and $35 \mathrm{~m} / \mathrm{s}$, and a decrease with the solids loading rate for the same velocities. He found that this decrease in the erosion rate is attributed to the cushioning effect promoted by inter-particle collisions. In order to improve the gas-solid flow in a lab-scale Circulating Fluidized Bed (CFB), riser Rossbach [9] used airfoil-shaped ring-type internals in a Computational Fluid Dynamics (CFD)-based design of experiments in which there are four variables considered: ring thickness, number of rings, spacing between rings and the insertion of a bottom ring. In this study, numerical simulations were performed with K-epsilon Turbulence Model and the Gidaspow Drag Model. The results of the simulation showed the case with a $45 \%$ decrease in the solids dispersion coefficient in comparison with the case without rings, which also requires $10 \mathrm{~mm}$ ring thickness and four rings. The case showed that the rings promote winding flow. Pawel [10] studied numerical simulation of a dense solid particle flow inside a cyclone separator. The two-phase gas-solid particles flow was modeled using a hybrid Euler-Lagrange approach, which accounts for the four-way coupling between phases. Compared to experimental data collected at the in-house experimental rig, the cyclone pressure drops evaluated numerically under-predicted the measured values. Deendarlianto [11] studied gas-solid two-phase flow via CFD. Simulations and experimental studies on the relevant phenomena in pipes were carried-out in the study. The pipes' diameters were 26 and $9.5 \mathrm{~m}$, respectively; Volume of Fluid (VoF) multiphase flow model was used in the simulation. Compared to the results in the experiment, a quantitative agreement between calculations and experimental data for the elongated bubble length and the time variation of the liquid hold-up are indicated.

Some researchers have also made efforts on the method and model in numerical simulation. Zhi [12], in Singapore, studied a new model based on Euler-Lagrange Equations, called Lagrangian algebraic slip mixture model. This model implements the contact between Euler model and the Lagrangian models. This new model was validated by comparing the experiment and simulation in the Two-Fluid model. M. Jafari et al. [13] studied the erosion rate of the horizontal pipe by two-phase flow analyses. They proposed a forecasting model for the pipeline erosion rate based on the discrete stochastic model. The simulation results and experimental data were compared, 
and particle impact angle and velocity were assessed. The new model can estimate erosion rates more accurately, especially in the gas and small particle flow. There are also lots of studies about the liquid film. Andriyanto Setyawan [14] studied circumferential liquid film distribution in annular two-phase flow, he used the conductance probe detected thickness distribution in an air-water horizontal annular two-phase flow. He found the thickness of the liquid film was influenced by the superficial liquid velocity. At the higher superficial gas velocity, a more uniform liquid film thickness distribution is commonly found. Moreover, compared to the available experimental data, the correlation has a good capability to predict the circumferential liquid film thickness distribution. Xie [15] studied droplet impact on a flowing liquid film in annular two-phase flow via simulation. A novel control volume finite element method with adaptive unstructured meshes was employed here to study three-dimensional droplet deposition process in the annular two-phase flow. The results in simulation agree with the experimental observations demonstrating the capability of the present method. Li [16] studied flow structure and flowed regime in large diameter pipes. In his study, adiabatic air-water two-phase flow experiments were been conducted to investigate flow regimes and their transitions. The Probability Density Function (PDF) and Cumulative Probability Density Function (CPDF) of area-averaged void fraction signals were utilised as the indicators for Self-Organised Neural Network (SONN) method to identify horizontal and vertical downward flow regimes, respectively. The results show that the flow regime maps agree well with that of $101.6 \mathrm{~mm}$, but do not agree well with that of smaller diameter pipes ( $25.4 \mathrm{~mm}$ and $50.8 \mathrm{~mm}$ ). A set of new transition criteria have been developed for downward flow regime transitions in large diameter pipes, which provide more accurate predictions for downward flow regime transitions in large diameter pipes. McCaslin [17] studied gravitational effects in horizontal annular liquid-gas flow in the simulation. There are three different sets of conditions in the annular and stratified-annular flow regimes in the simulation. A simple model is developed that helps to characterise the dynamics of the liquid annulus and aids in understanding the effect of secondary gas flow on the circumferential motion of the film. Aliyu et al. [18] studied interfacial friction in upward annular gas-liquid two-phase flow in pipes. The experiments were carried out in a large diameter flow loop of $101.6 \mathrm{~mm}$ internal diameter with the superficial gas and liquid ranges of 11-29 m/s and $0.1-1.0 \mathrm{~m} / \mathrm{s}$ respectively. Significant discrepancies were found between many of the published correlations and the large pipe data, primarily in the thick film region at low interfacial shear stress. Andriyanto [19] studied the effect of the fluid properties on the wave velocity and wave frequency in annular pipe. He used water and glycerol solution to analyze the influence of the viscosity, and water and butanol solution to analyze the influence of the surface tension. The experiments summarized the influence of fluid property like viscosity and surface tension to the gas-liquid flow in pipes.

Although the above has laid a good foundation for the studies in gas-solid pipe flow, the flow conditions in annular pipes have not been thoroughly investigated. Particularly, the flow in the region of the drill bit, where the carrier medium air exits the inner pipe and flows into the annular channel is still not clear. Moreover, with the solids added into the flow, the condition becomes complicated. Therefore, in this study, we intend to describe the flow characteristics of the solids in the annulus section to a certain degree of detail. This is to be carried out by both CFD simulations and Electrical Capacitance Tomography (ECT) measurement. Such information could be valuable for the industry. Also, we wish to verify the simulation methods and results by ECT measurement, which could establish a certain degree of guide or experience for future design of the trilling process and equipment development.

\section{Physical Model and Simulation Set-Up}

As shown in Figure 1, horizontal drilling includes a horizontal section and an inclined section. This can be modelled by an annulus structure with an inner pipe supplying air as the carrier fluid to bring out the debris in the annular space. Attached at the end of the inner pipe is the drill bit, where air exits the inner pipe and flows into the annulus space. This is also the place where the solid particles 
enter the annulus space and are carried away by air; thus, it can be regarded as the inlet boundary for our numerical simulation to be introduced in the subsequent sections.

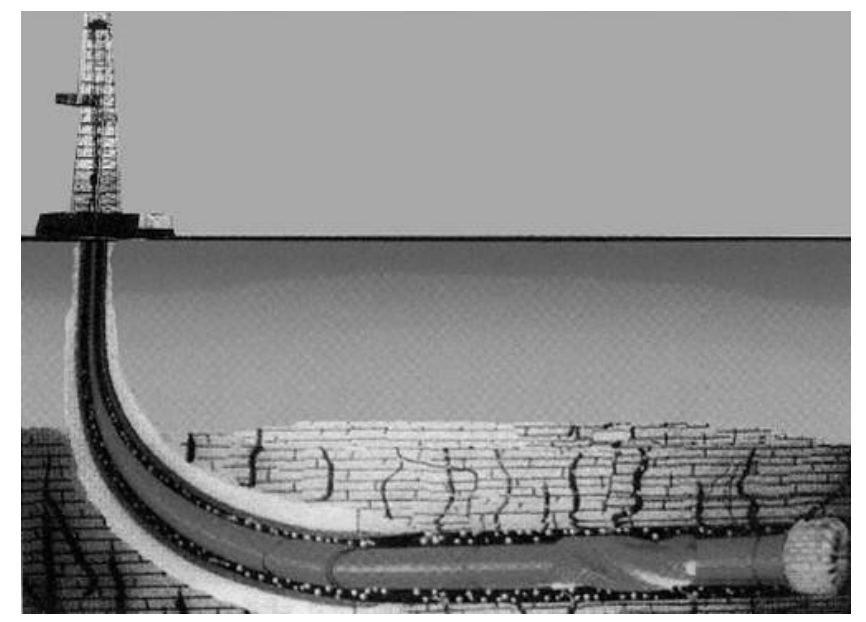

Figure 1. Horizontal drilling.

To simulate the flow in the annulus space, GAMBIT software is used to create the computation domain, shown in Figure 2, and to generate the model and mesh for Fluent calculation. The real configuration is modelled as a set of two coaxial pipes of different diameters, the inner pipe and the outer pipe. The carrier fluid, namely air, enters the inner pipe as indicated as $A$ in Figure 2, and exits the inner pipe at the other end as indicated as $B$. The axial distance between the inner and outer pipe at the end $B$ is $100 \mathrm{~mm}$. The left end of the outer pipe is blocked, representing the rock/earth being drilled. On exiting the inner pipe at point $B$, the carrier fluid will take a U-turn to enter the annulus space, as indicated by the arrows. Solid particles will flow into the annular pipe at the point marked by "Solid inlet" and be carried away by the air flow. The solid inlet is a circle of $10 \mathrm{~mm}$ in diameter, in which the center is located at the height of $115.5 \mathrm{~mm}$ on the left end of the outer pipe. The inner pipe is $500 \mathrm{~mm}$ in length and $75 \mathrm{~mm}$ in diameter, while the outer pipe is $520 \mathrm{~mm}$ in length and $156 \mathrm{~mm}$ as the inner diameter.

A computational grid is built using a combination of Cooper and TGrid, as shown in Figure 3 as an example for the U-turn region. The number of a computational element is $746,847$.

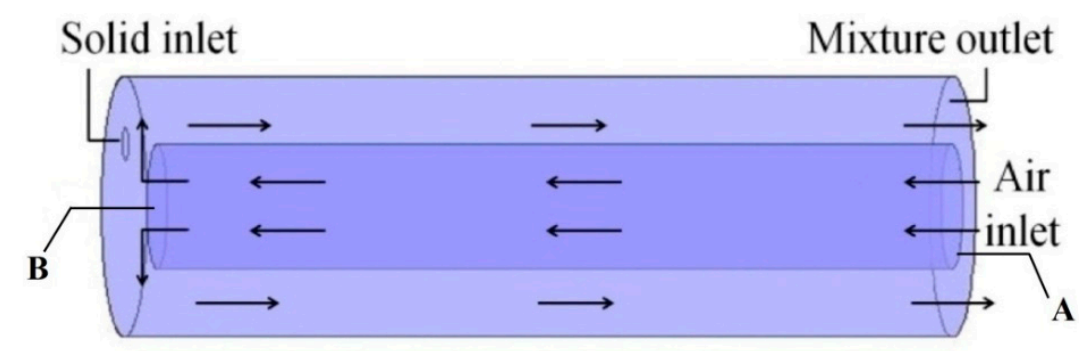

Figure 2. Structure of annular pipe. 


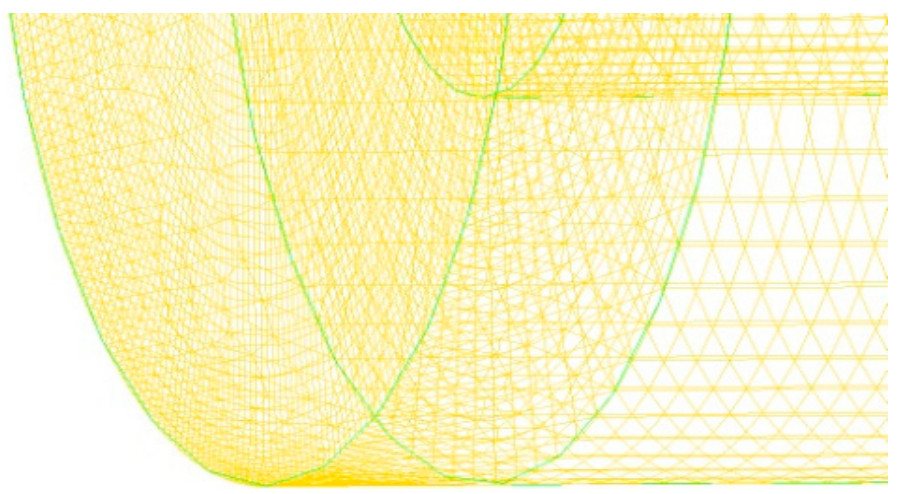

(a)

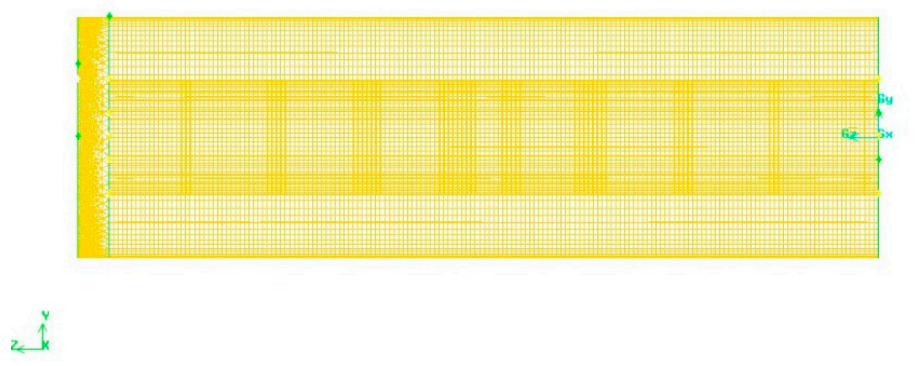

(b)

Figure 3. (a) Meshing in annular space; (b) Cross section of meshing in annular space.

\section{Numerical Simulation and Analysis}

Discrete Phase Model (DPM) is used to calculate the particle motion in the annular space. DPM is based on a Euler-Lagrangian framework. In this model, the fluid is a continuous medium, and the particle phase is discrete. The particles are categorised into two groups. One is characterised by instantaneous collision movement driven by momentum and the other by suspension movement controlled by the fluid drag force. Thereby a particle motion decomposition model is established [12]. In this model, the movement of the fluid is described by N-S equations of a continuous medium. Particle motion is obtained by calculating the trajectories of every particle. The trajectory of the particle can be calculated by the following formula,

$$
\frac{d u_{p}}{d t}=F_{D}\left(u-u_{p}\right)+\frac{g_{x}\left(\rho_{p}-\rho\right)}{\rho_{p}}+F_{x}
$$

where $u_{p}$ is the velocity of particle, $u$ is the velocity of fluid, $\rho_{p}$ is density of the particle, $\rho$ is the density of the fluid, and $g_{x}$ is the gravity of the particle. The first item on the right side of the equal sign is the drag of the unit mass particle, the second item is the resultant force of gravity and buoyant force, and the third item is the resultant force of other additional forces. And $F_{D}$ is calculated by using the following formula,

$$
F_{D}=\frac{3 \mu C_{D} R e_{p}}{4 \rho_{p} d_{p}^{2}}
$$

The drag force item in the formula is obtained by the correlation proposed by Moris et al. [20],

$$
C_{D}=a_{1}+\frac{a_{2}}{R e_{p}}+\frac{a_{3}}{R e_{p}^{2}}
$$

where $a_{1}, a_{2}, a_{3}$ are constants, $R e_{p}$ is Reynolds number of particle. 
For the coupling between the discrete phase and the continuous phase, there is mainly the particle cloud model and the stochastic particle model proposed by Gosman et al. [21]. The second model is used by most researchers, which is also used in this paper.

In addition to DPM, the Two-Fluid model is commonly used for multiphase flows. The Two-Fluid model is based on the Euler framework. Each phase will be seen as a continuous medium. Each of the two phases flow with different characters and can penetrate into the other, and the forces are coupled. A set of equations contain momentum equations and a continuity equation [22]. The continuity equation for the fluid phase is,

$$
\frac{\partial}{\partial t}\left(\alpha_{q} \rho_{q}\right)+\nabla \cdot\left(\alpha_{q} \rho_{q} \overrightarrow{v_{q}}\right)=\sum_{p=1}^{n} \dot{m}_{p q}
$$

where $\alpha_{q}$ is the volume fraction of phase $q, \rho_{q}$ is the physical density of phase $q, \overrightarrow{v_{q}}$ is the speed of phase $q$, and $\dot{m}_{p q}$ is the mass transfer flux from phase $p$ to phase $q$.

Moreover, the momentum conservation equation is,

$$
\frac{\partial}{\partial t}\left(\alpha_{q} \rho_{q} \vec{v}_{q}\right)+\nabla \cdot\left(\alpha_{q} \rho_{q} \vec{v}_{q} \vec{v}_{q}\right)=-\alpha_{q} \nabla p+\nabla \cdot \overline{\bar{\tau}}_{q}+\sum_{p=1}^{n}\left(\vec{R}_{p q}+\dot{m}_{p q} \vec{v}_{p q}\right)+\alpha_{q} \rho_{q}\left(\vec{F}_{q}+\vec{F}_{l i f t, q}+\vec{F}_{V m, q}\right)
$$

where $\overline{\bar{\tau}}_{q}$ is pressure strain tensor of phase $q, \vec{F}_{q}$ is the external volume force, $\vec{F}_{l i f t, q}$ is the lift force, $\vec{F}_{V m, q}$ is the virtual mass force, $\vec{R}_{p q}$ is the interaction force between phases, $p$ is pressure shared with all phases, and $\vec{v}_{p q}$ is the speed difference between the two phases. Standard $k-\varepsilon$ model is used throughout the simulation. The wall boundary conditions in this paper are non-slip conditions. In the large-scale flow field analysis, the velocity of the fluid near the wall surface has little effect on the flow field, while the medium with low viscosity has low shear force, such as air, which can be neglected. Slip conditions are only used in flow field analysis at microscopic scales or in analysis of special cases with a high viscosity medium [23].

To begin with, air inlet speed is set to $1.5 \mathrm{~m} / \mathrm{s}$, and all the walls are assigned no-slip conditions. The outlet of the annular channel is set to the ambient pressure. Contour and vector maps of the velocity are shown in Figures 4 and 5 respectively. It can be seen that the speed of air at the exit of the inner pipe becomes faster due to the change of pressure and geometric change. Swirls can be seen in the early stage of flow in annular space. That contributes to the decrease of speed value and the flow keeps relatively steady in following stage.

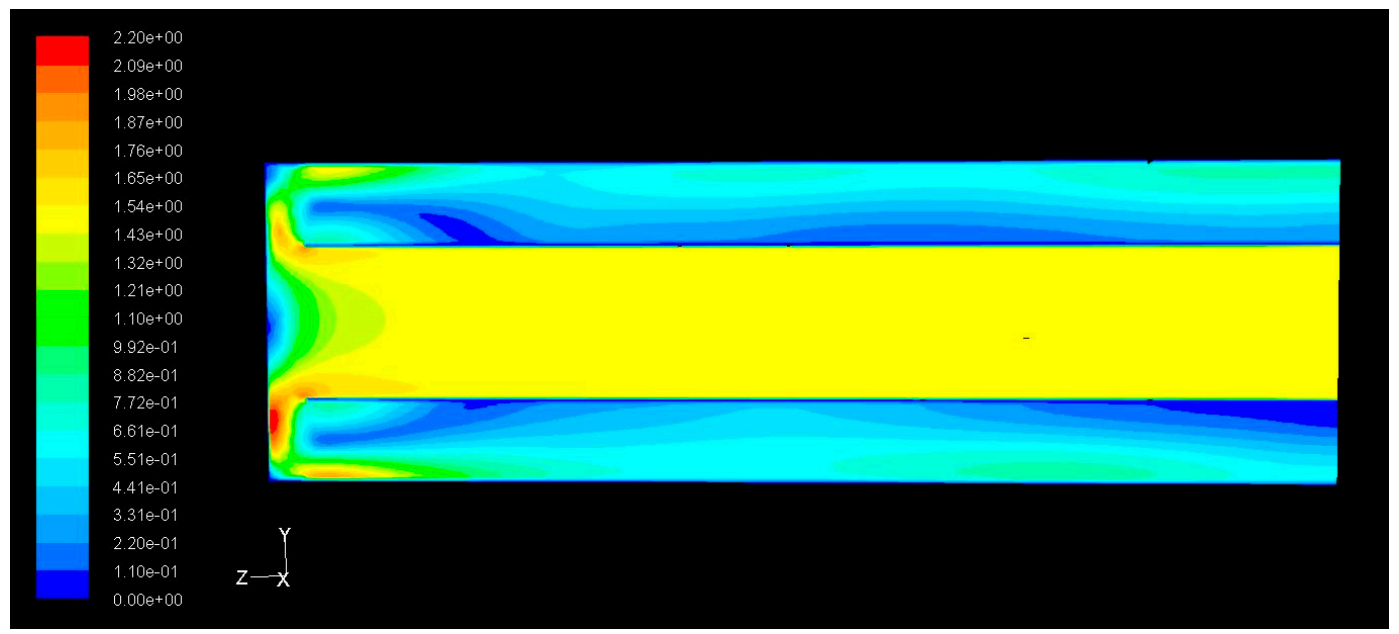

(a)

Figure 4. Cont. 


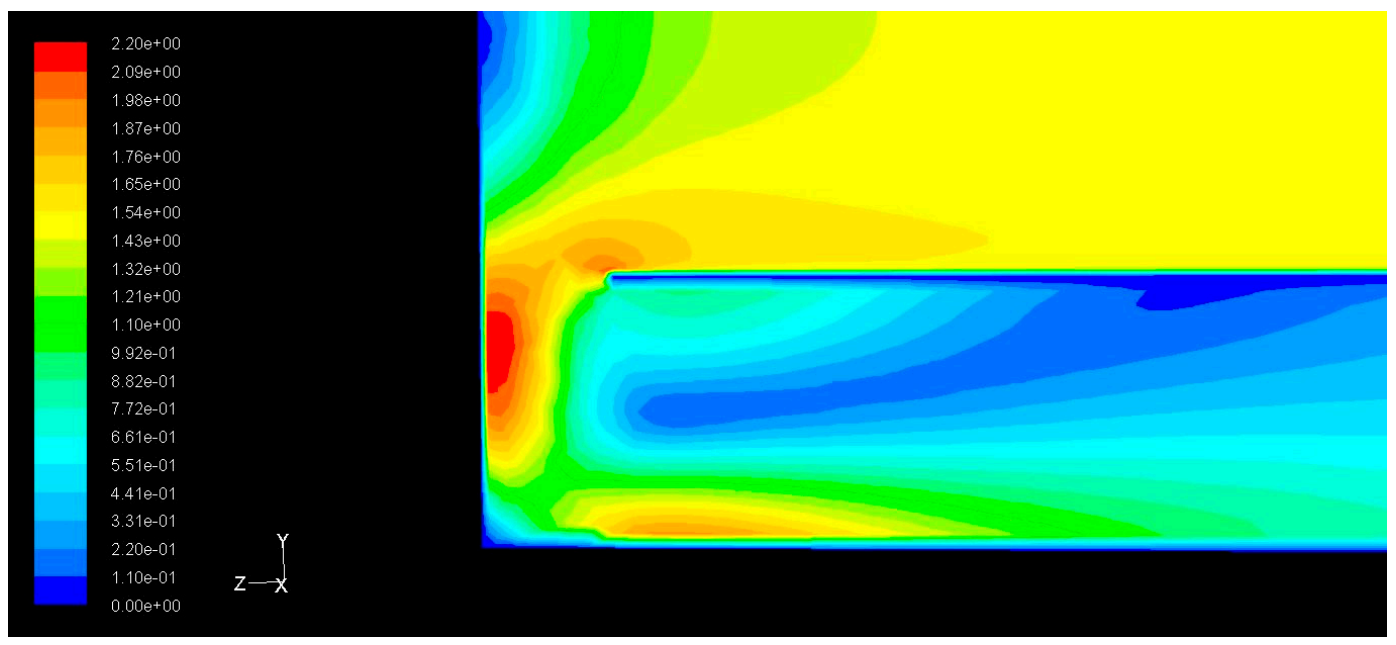

(b)

Figure 4. (a) Contour of the main airflow field; (b) Contour of partial enlarged detail in main airflow field.

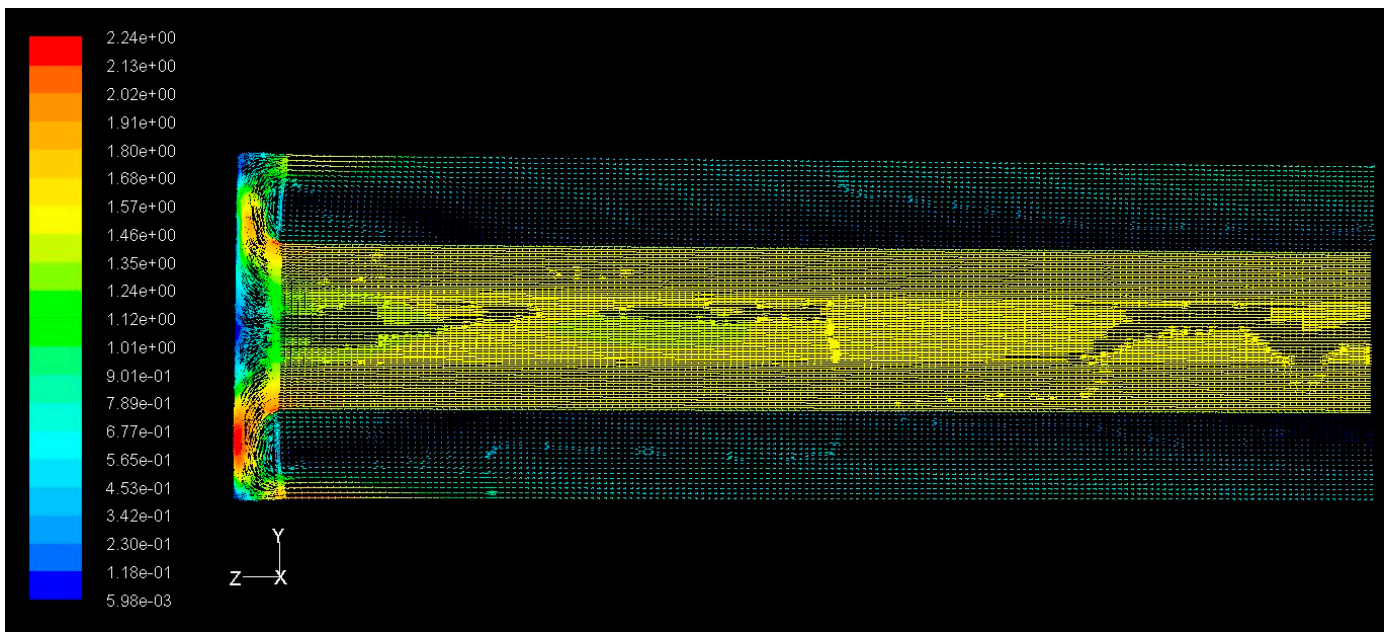

(a)

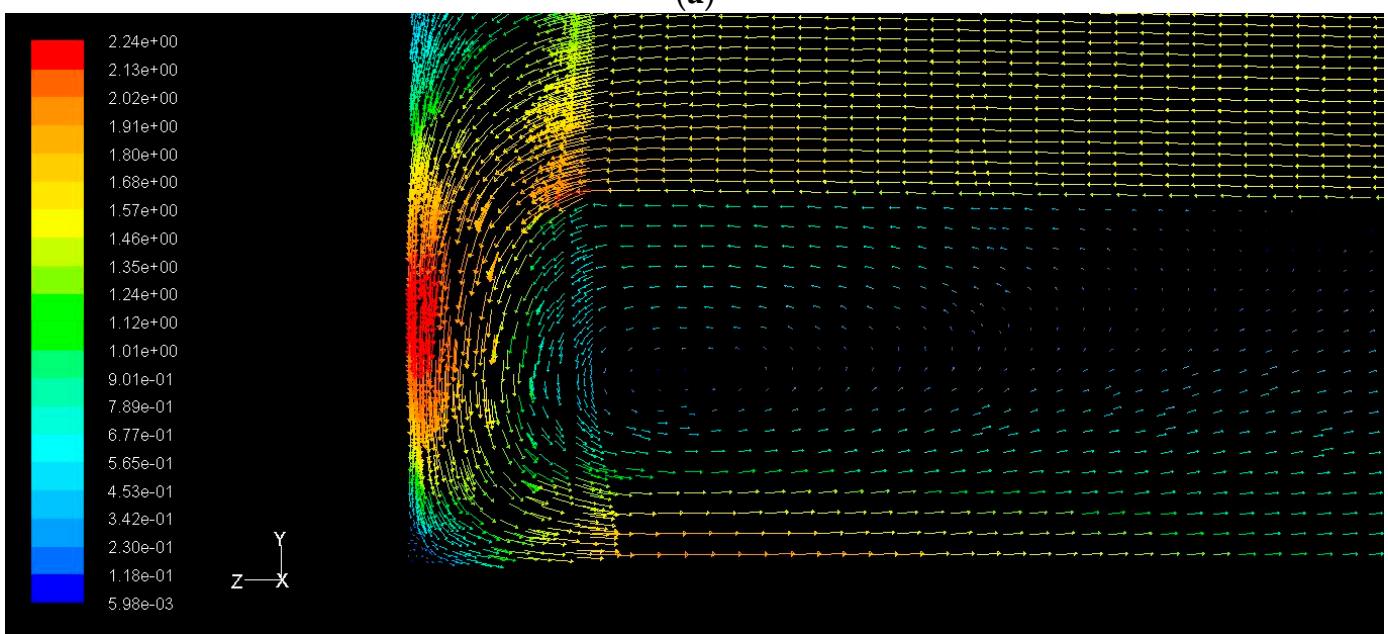

(b)

Figure 5. (a) Vector map of the main airflow field; (b) Vector map of partial enlarged detail in main airflow field. 
To explore two-phase flow in the annular channel, three series of simulations are carried out. The first two sets use DPM to simulate the horizontal annulus section, and the third one uses the Two-Fluid model to simulate the particle motion in the inclined section.

In the first series of simulations, silicon dioxide with the density of $2600 \mathrm{~kg} / \mathrm{m}^{3}$ is used for the solid particles with a range of diameters from $0.1 \mathrm{~mm}$ to $0.4 \mathrm{~mm}$. The particles enter the annular section from the point "Solids inlet" indicated in Figure 2. Air velocity of $1.5 \mathrm{~m} / \mathrm{s}$ is assigned at the entrance A of the inner pipe, as shown in Figure 2. The simulation results are plotted in Figure 6

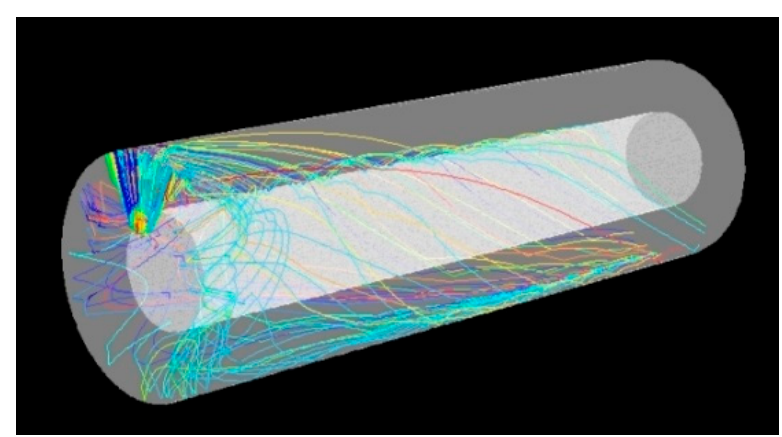

(a)

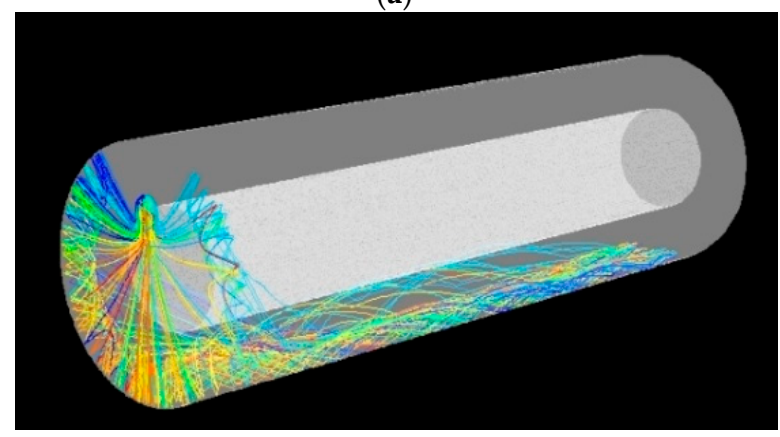

(b)

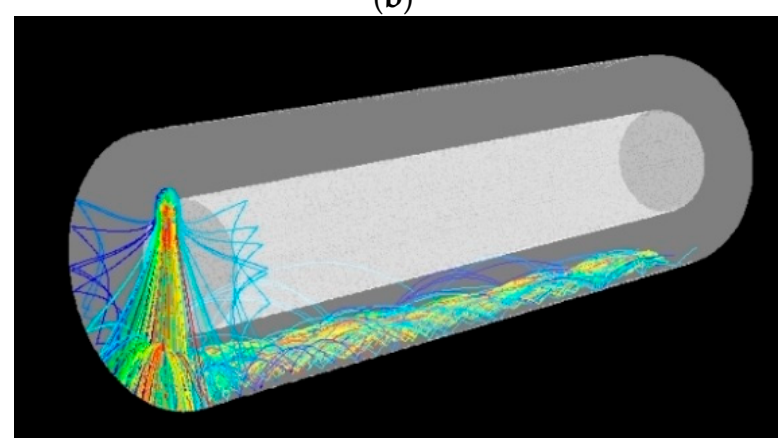

(c)

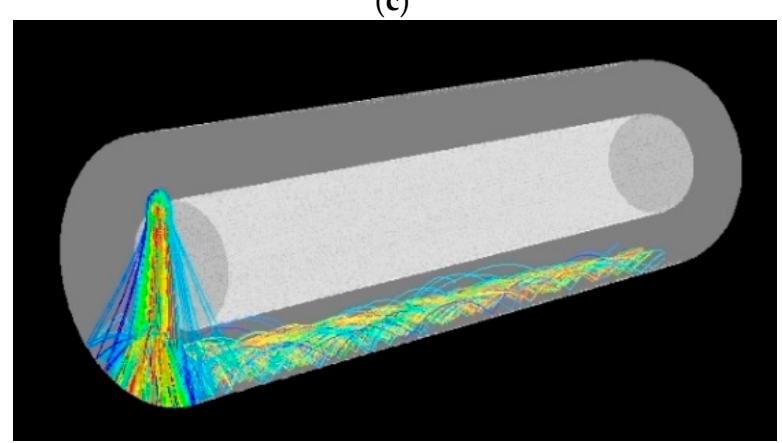

(d)

Figure 6. Trajectories of particles with different diameters: (a) Particle diameter is $0.1 \mathrm{~mm}$; (b) Particle diameter is $0.2 \mathrm{~mm}$; (c) Particle diameter is $0.3 \mathrm{~mm}$; (d) Particle diameter is $0.4 \mathrm{~mm}$. 
It can be seen in Figure 6a that with a diameter of $0.1 \mathrm{~mm}$, the particles are distinctively dispersed. The trajectories of the particles demonstrate a swirling flow around the inner pipe. Noticeably, some particles move upward from the solid inlet and stay suspended for a certain length in the upper part of the annular channel. This may be due to the significant vertical component of the air velocity in this region. As the vertical airspeed diminishes downstream, the gravitational effect becomes more pronounced, and the particles gradually move downward. With increased particle diameter, the effect of the airlift of the particles becomes weaker. The particles will move gradually towards the bottom of the pipe. Therefore, the particles with $0.4 \mathrm{~mm}$ diameter are more densely packed on the bottom of the pipe.

The second series of simulations explore the effects of different air velocities on the flow patterns of the particles. As shown in Figure 7, when the particle diameter is $0.2 \mathrm{~mm}$, with the increase of the air inlet velocity, the particles in the inlet wall will encounter a stronger air drag force. Additionally, the particles spread from the centre to the edges. Because there is no change in the position of the particle entrance, the higher the air velocity is, the wider the solids spread. After the particles enter the annular channel, they also tend to flow towards lower positions due to the diminishing vertical component of the air velocity.

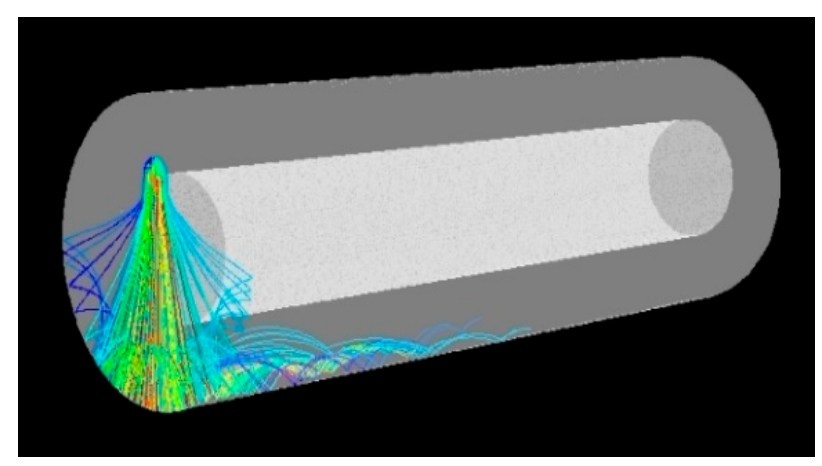

(a)

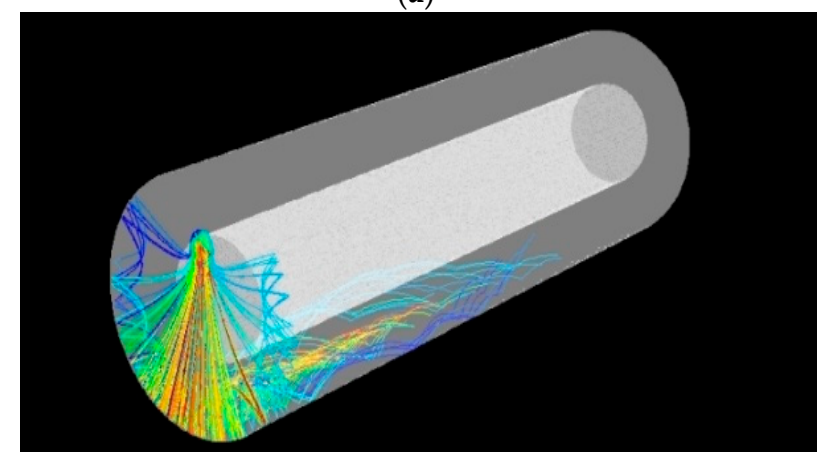

(b)

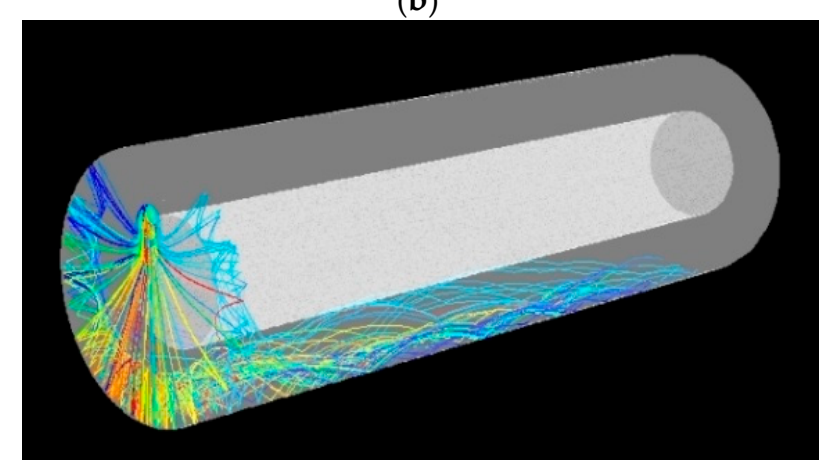

(c)

Figure 7. Cont. 


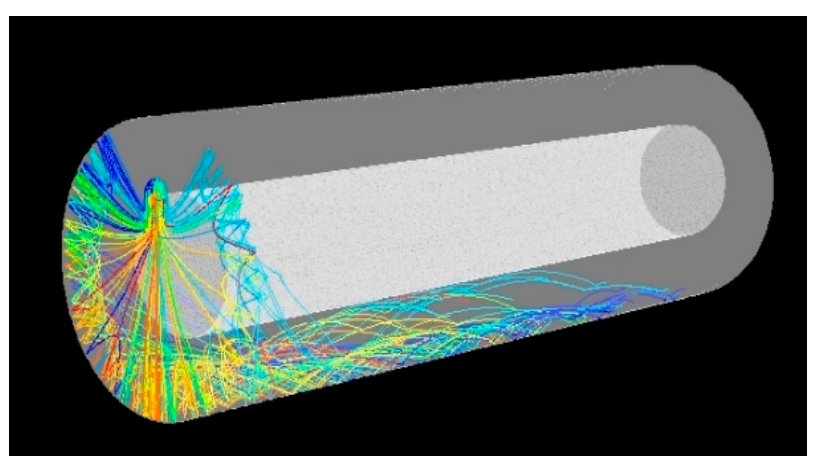

(d)

Figure 7. Trajectories of particles at different air velocity: (a) Air velocity is $2 \mathrm{~m} / \mathrm{s}$; (b) Air velocity is $2.5 \mathrm{~m} / \mathrm{s}$; (c) Air velocity is $3 \mathrm{~m} / \mathrm{s}$; (d) Air velocity is $3.5 \mathrm{~m} / \mathrm{s}$.

It will give us a more complete view of the flow if we take a look at the solid distributions in the cross-sections of the pipe. For this purpose, the data from the second series of the simulation are plotted in Figure 8. The plot corresponds to a cross section $5 \mathrm{~mm}$ from the feed point of the solid particles, where the cross-section is divided into four horizontal sections. Then, the figure plots the number of particles in each horizontal section divided by the area of the section. Thus a particle number density per unit area is obtained. It can be seen in the figure that in general the number density decreases with the height from the bottom of the pipe. Also, as the air inlet velocity increases, more particles can be carried into higher positions, indicating that larger air velocity will be capable of transporting more solid particles.

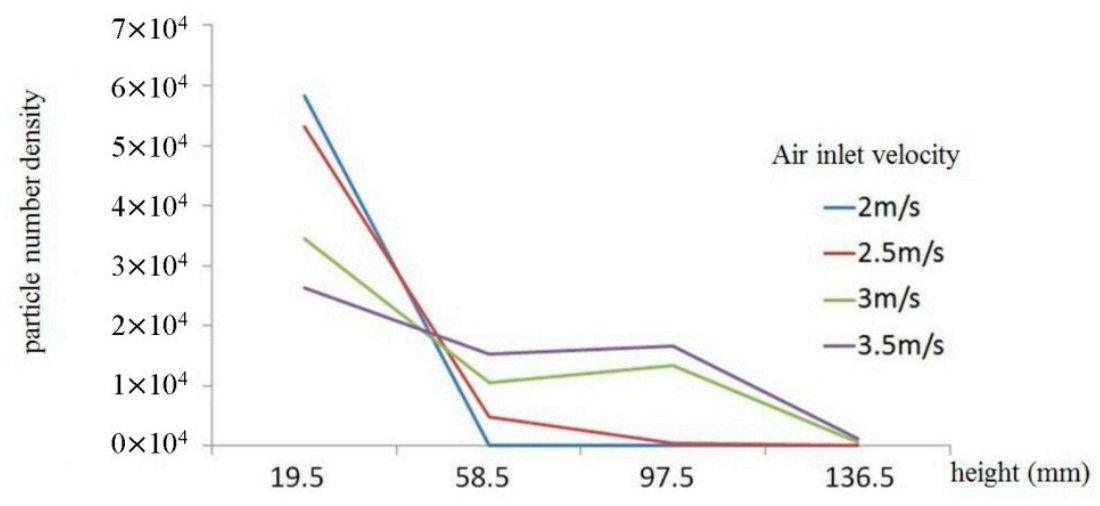

Figure 8. Curve of particle number density.

The third series of simulations explore the fluid flow under gravitational force in the inclined section, in order to understand the backflow of sand due to possible stoppage of air flow for various reasons such as system malfunction. The Two-Fluid model is applied for its suitability to dense flows. The cross-section $z=100 \mathrm{~mm}$ in the annular space is selected to observe the medium distribution. Moreover, the velocity of the solid phase at the bottom of the annular section is calculated. The mass flow rate of the solid phase is set to $0.3 \mathrm{~kg} / \mathrm{s}$; the material is silicon dioxide and the pipe has an incline of 45 degrees. As shown in Figure 9, solid particles slide on the bottom of the inclined section. The solid distribution at the observed cross-section indicates that the solid concentration at the bottom of the pipe is relatively high, shown in Figure 10. Moreover, the speed at point M is shown in Figure 11, which is $0.532 \mathrm{~m} / \mathrm{s}$. 


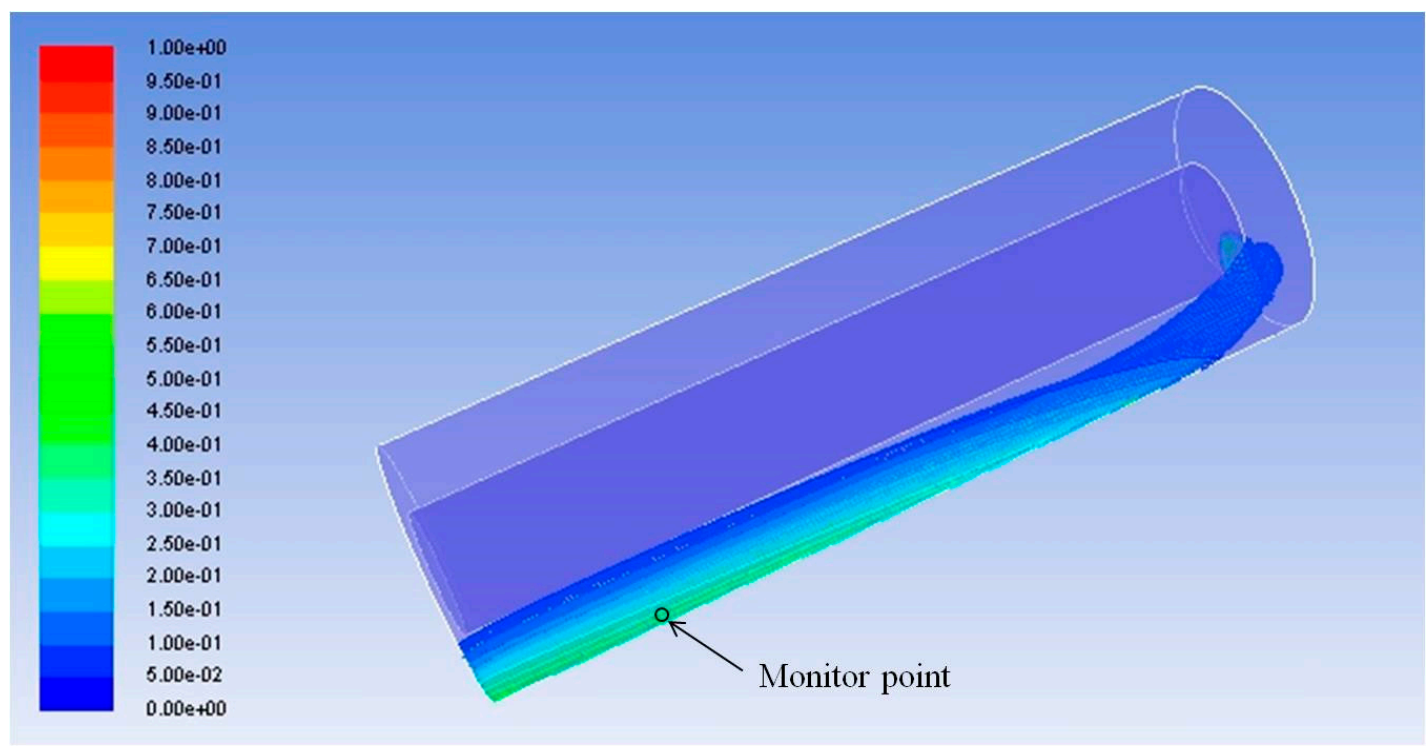

Figure 9. Solid concentration in Two-Fluid model.
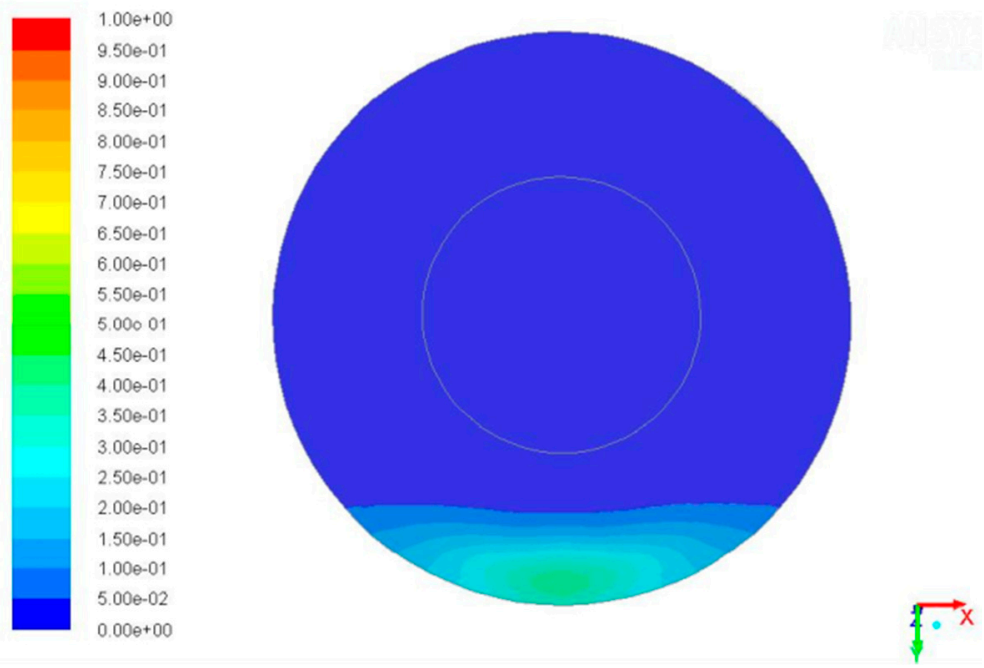

Figure 10. Solid concentration of the cross-section $\mathrm{z}=100 \mathrm{~mm}$.

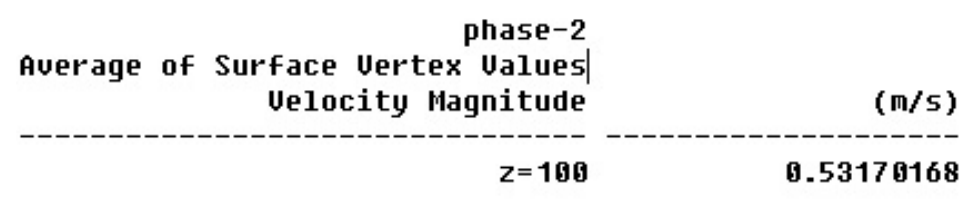

Figure 11. Final stable velocity at the monitor point.

\section{Experimental Procedure}

To verify the CFD simulations for the inclined section, an experimental rig is built to measure the distribution and speed of the solid particles simultaneously. The rig is shown in Figure 12 in which the ECT sensor and the speed sensor are respectively arranged on the inner and outer concentric pipes. The ECT system is on the right and the velocity detection system on the left. 


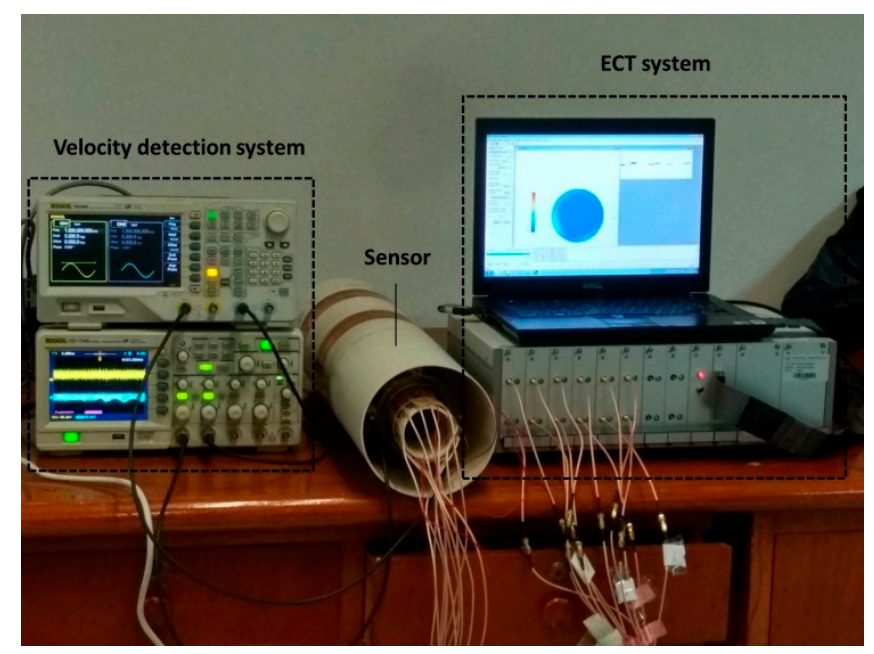

Figure 12. Experimental system.

\subsection{ECT Experiments}

The mechanism of ECT technology is that changes in the shape and distribution of multiphase flow or environmental parameters, such as temperature and humanity, contribute to the changes in dielectric constant. Then, the measured capacitance value between each pair of electrodes will follow the variation. Therefore, it is feasible to reconstruct the image of multiphase flow distribution via some reconstruction algorithm according to the capacitance value. Consequently, the solid phase reflects the high grey value of the image, which manifests that the image can reflect the distribution of the medium.

Based on electrical capacitance tomography technology, the sensor has been arranged on the outer wall of the inner tube to monitor the medium distribution of the annular space. Characteristics in Electrical Capacitance Tomography (ECT) experiments are shown in Table 1. As shown in Figure 13, the sensor consists of detection electrodes, shielding electrodes and covers. The middle of the two electrode pairs for velocity measurement is at the same position with the imaging sensor in the axial direction. Moreover, it is the same position with the point $M$ in simulation 3 which is at the distance of $400 \mathrm{~mm}$ to the solid phase inlet. As shown in Figure 13, twelve detection electrodes are arranged averagely, and the shielding electrodes are arranged between the detection electrodes in the radial direction as well as the upper and lower area in the axial direction. The whole sensor part is surrounded by the shielding cover. The whole shielding part is grounded to prevent inter-signal interference and external interference. Ang_d is $20^{\circ}$, and Ang_s is $8^{\circ}$. $H \_d$ is $90 \mathrm{~mm}$.

Table 1. Characteristics in Electrical Capacitance Tomography (ECT) experiments.

\begin{tabular}{cc}
\hline Experimental Characteristics & Description \\
\hline$M$ & The point to monitor speed \\
$A n g \_d$ & The angle of each detection electrode width \\
$A n g \_s$ & The angle of each radial shielding electrode width \\
$H \_d$ & The height of detection electrode \\
\hline
\end{tabular}




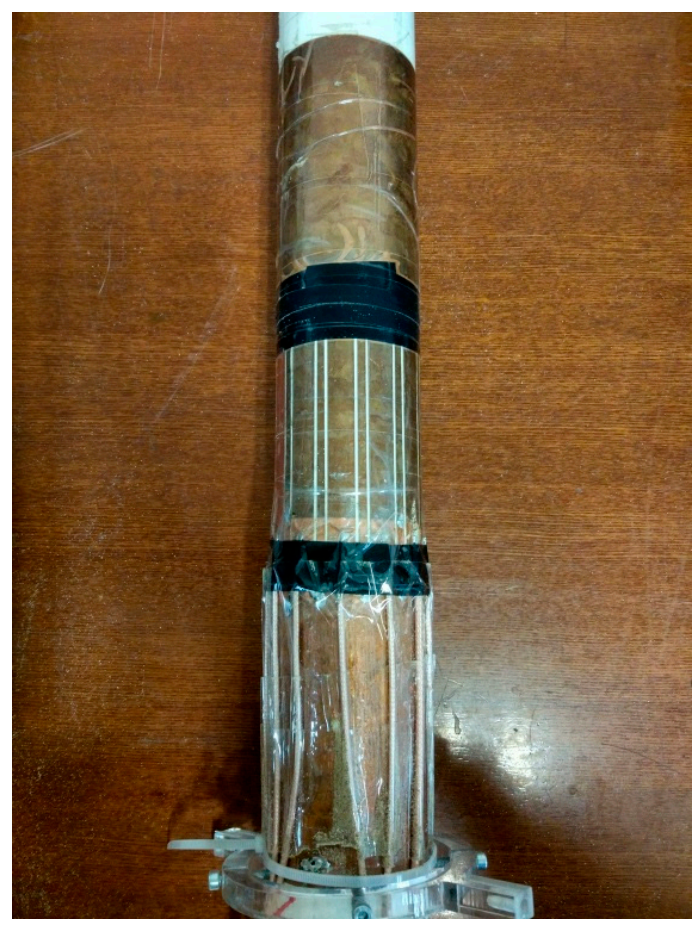

Figure 13. Structure of the sensor.

To verify the feasibility of this structure of the sensor in detecting the annular space, the imaging has been simulated by ANSOFT software. The same solid phase distribution as the cross-section in simulation 3 in CFD is set up, as shown in Figure 14a. The reconstructed image is shown in Figure 14b after the calculation, and the distribution of the solid part in the image is corresponding to the actual situation. The feasibility of this sensor structure to detect annular space is verified. The experimental results are discussed in the following section.

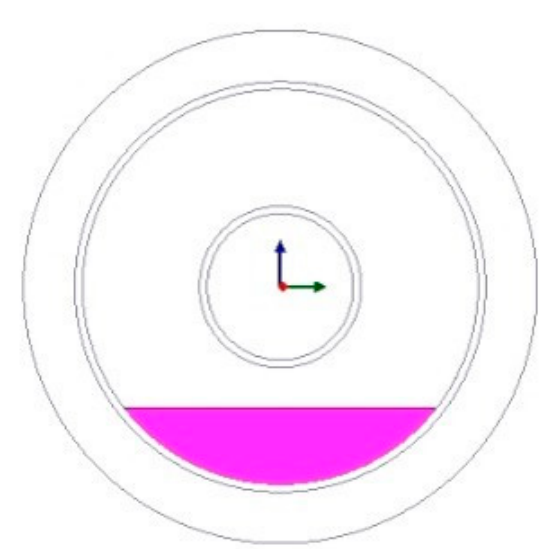

(a)

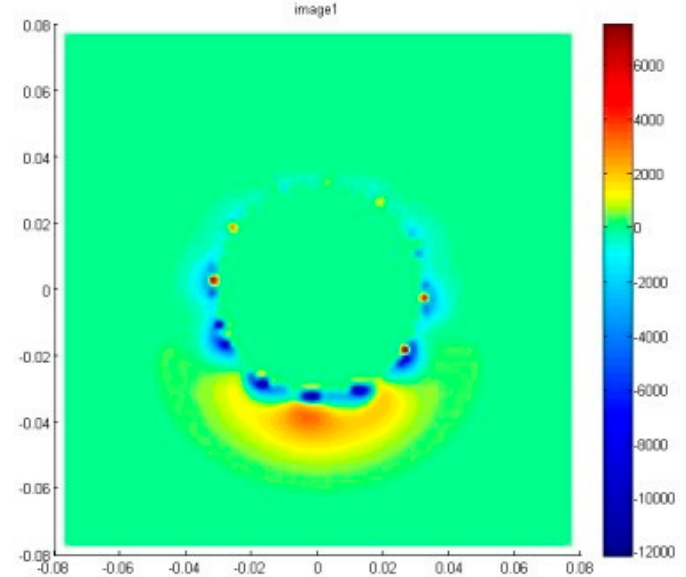

(b)

Figure 14. Simulation: (a) True distribution, (b) Reconstructed solids distribution.

When a lump of solid passes through the bottom of the channel, the solid distribution can be reconstructed via ECT system. As indicated in Figure 15, the grey value presents relatively high in the position of solid, while the value in the rest is small. The results agree well with the actual condition. 


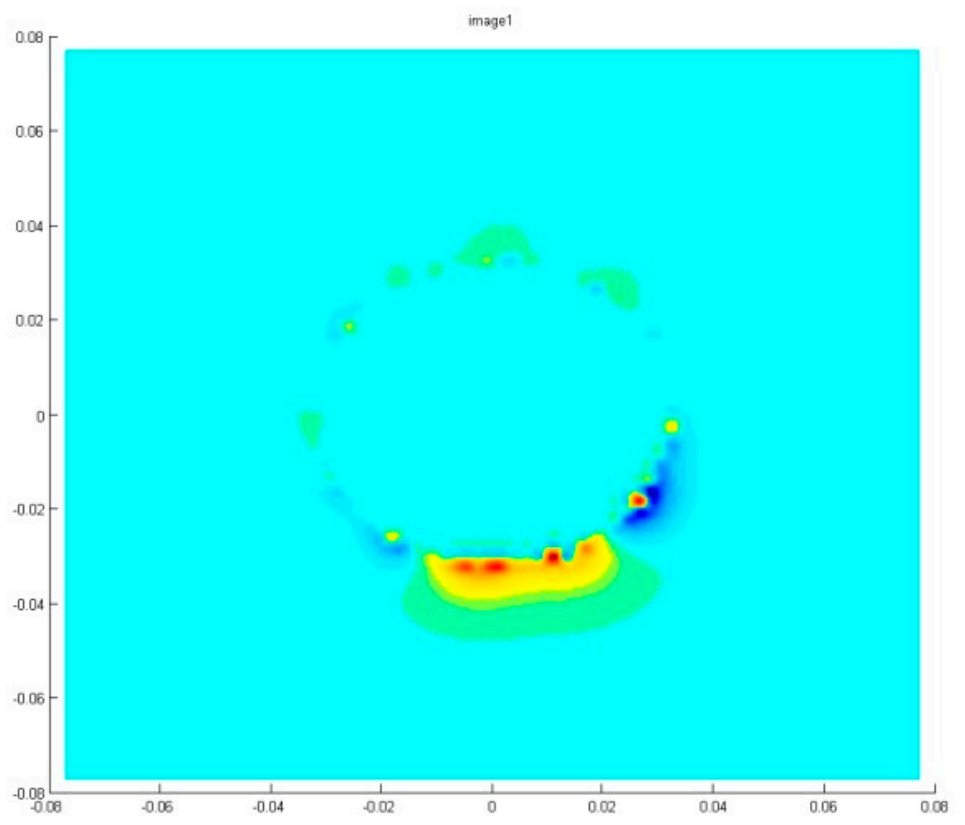

Figure 15. Reconstructed solids distribution.

\subsection{Speed Detection Experiments}

To investigate the velocity of the solid phase in the channels simultaneously, the velocity sensor electrode is arranged on the inner wall of the outer pipe. The rig is shown in the left section of Figure 12, in which the inner pipe and the outer pipe can be seen, together with a function generation and an oscilloscope. Electrical sensors are used to measure the speed of the solids. Basically, a sensor is structured like a pair of combs of thin electrodes, with the electrodes interlaced to form a capacitor, shown in Figure 16. During the measurement, one set of electrodes is charged by the signal from the function generator, and the other set of electrodes receive signals. When a lump of solids flow past the electrodes, the set of receiving electrodes will produce a pulse of signal that will be recorded by the oscilloscope.

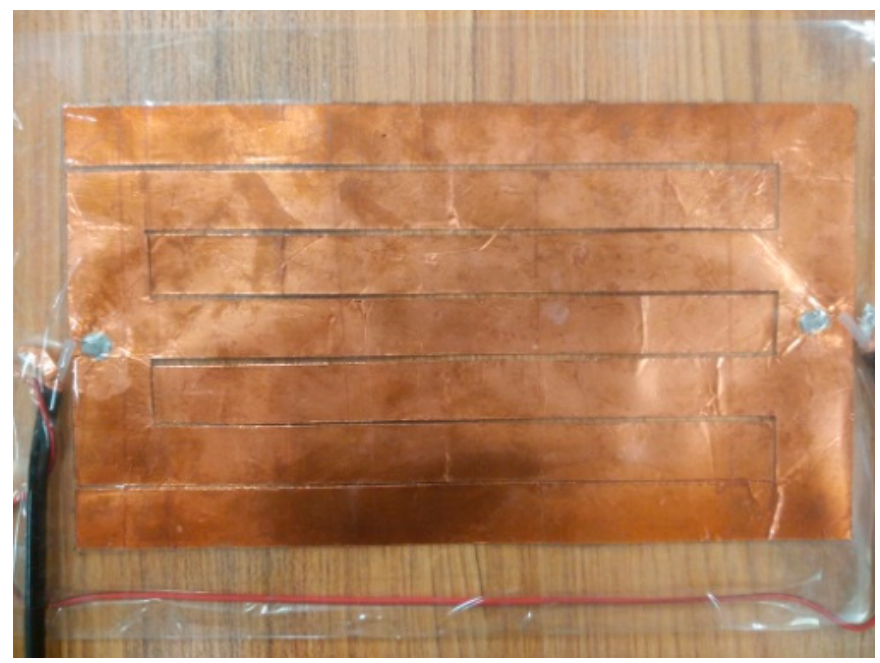

Figure 16. An electrode pair for measurement.

Experimental characteristics in speed detection are shown in Table 2. Two sensors $E_{-} A$ and $E_{-} B$ are mounted on the pipe wall in the inclined section. The upstream sensor is marked as $E_{-} A$, and the 
downstream sensor is marked as $E \_B$. The two sensors are separated by $D$ of $30 \mathrm{~cm}$, as indicated in Figure 17. The signals will be collected continuously during an experiment. Thus, two series of curves of signals will be recorded from the two sensors. When a solid lump flows past the two sensors, ideally a peak will appear in each of the recorded curves. The two peaks will be separated by a time interval as the solid lump passes the upstream and the downstream sensors at a different time.

Table 2. Experimental characteristics in speed detection.

\begin{tabular}{cc}
\hline Experimental Characteristics & Description \\
\hline$E \_A$ & The upstream electrode pair \\
$E \_B$ & The downstream electrode pair \\
$D$ & Distance between two sensors \\
$V o l$ & The voltage of the signal \\
$T u$ & Time Unit \\
$V \_e$ & The velocity of lump obtained in the experiment \\
$V \_s$ & The velocity of lump obtained in the simulation \\
\hline
\end{tabular}

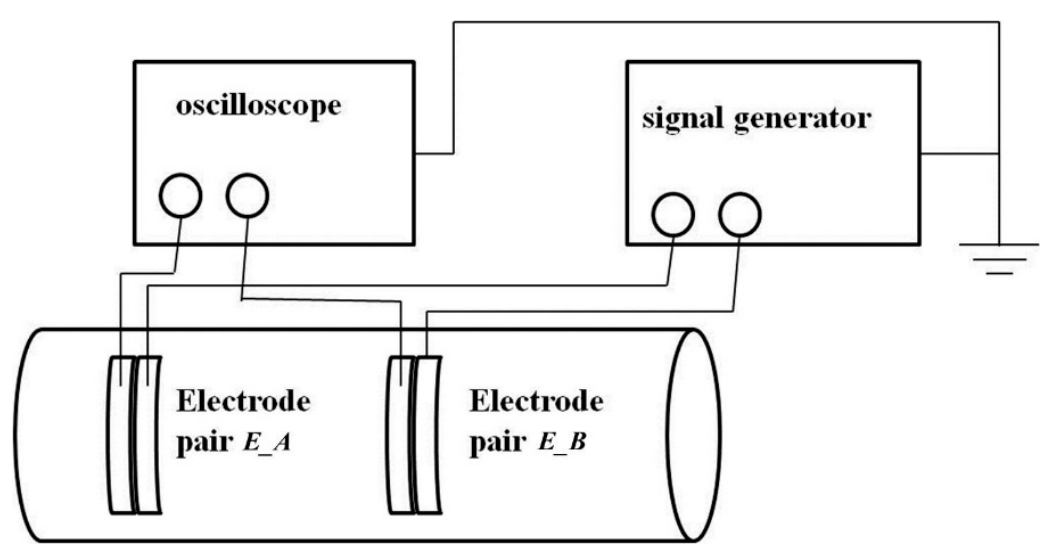

Figure 17. Schematic of velocity measured with the method of cross-correlation.

In the experiment, the sine wave of $100 \mathrm{kHz}$ is used to charge the electrodes, and $\mathrm{Vol}$ is $5 \mathrm{~V}$. The waveform is recorded by an oscilloscope, whose $T \_u$ is set to $200 \mathrm{~ms}$. After the signals are recorded, the speed of the solids is calculated using cross-correlation method, which indicates the correlation between the two-time series of signals.

$$
R(n)=(1 / N) \sum[x(m) y(m+n)]
$$

In the above formula, $R(n)$ is the cross-correlation coefficient, $n$ is the shifting number of two series, and $\mathrm{m}$ is the number index of the signals ranging from 0 to $N-1$. In the experiment, the pipes are inclined at 45 degrees, and a lump of sand is poured into the annular space from one end. Then, the flow velocity is obtained from signal processing using the cross-correlation method in Matlab software.

Figure 18 shows the signals received from sensor $E_{-} A$, in green colour, and from sensor $E_{-} B$, in red colour. Peak values on each curve can be seen. Also, it can be seen that the range of the peak values on the green curve is slightly broader than on the red curve, indicating that a solid lump spends more time in sensor $E \_A$, or that the speed of the solid lump in sensor $E \_B$ is faster than in sensor $E \_A$. This is reasonable, because of the acceleration the solid lump should flow faster in the downstream lower position, i.e., near sensor $E \_B$. According to the data from the oscilloscope, the correlation coefficient of the two waveforms is calculated and shown in Figure 19, which corresponds to $V \_e$ of $0.517 \mathrm{~m} / \mathrm{s}$. 


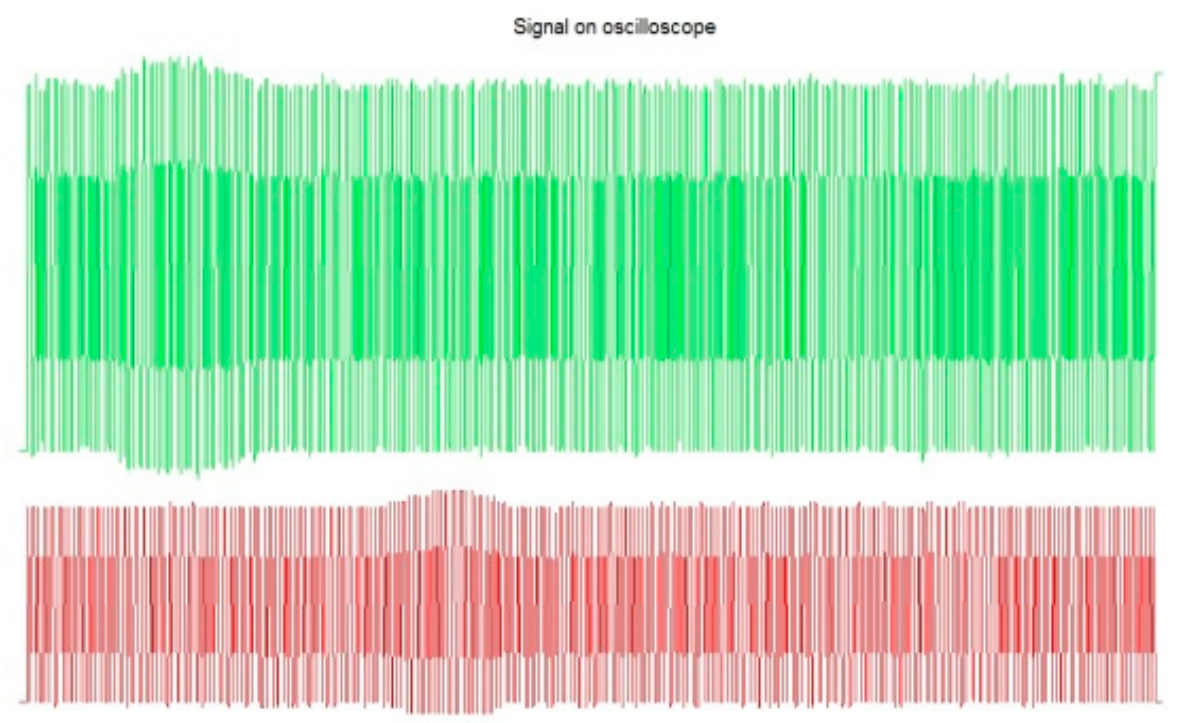

Figure 18. Signal on the oscilloscope (the green signal is received from sensor $E \_A$, the red signal is received from sensor $E \_B$ ).

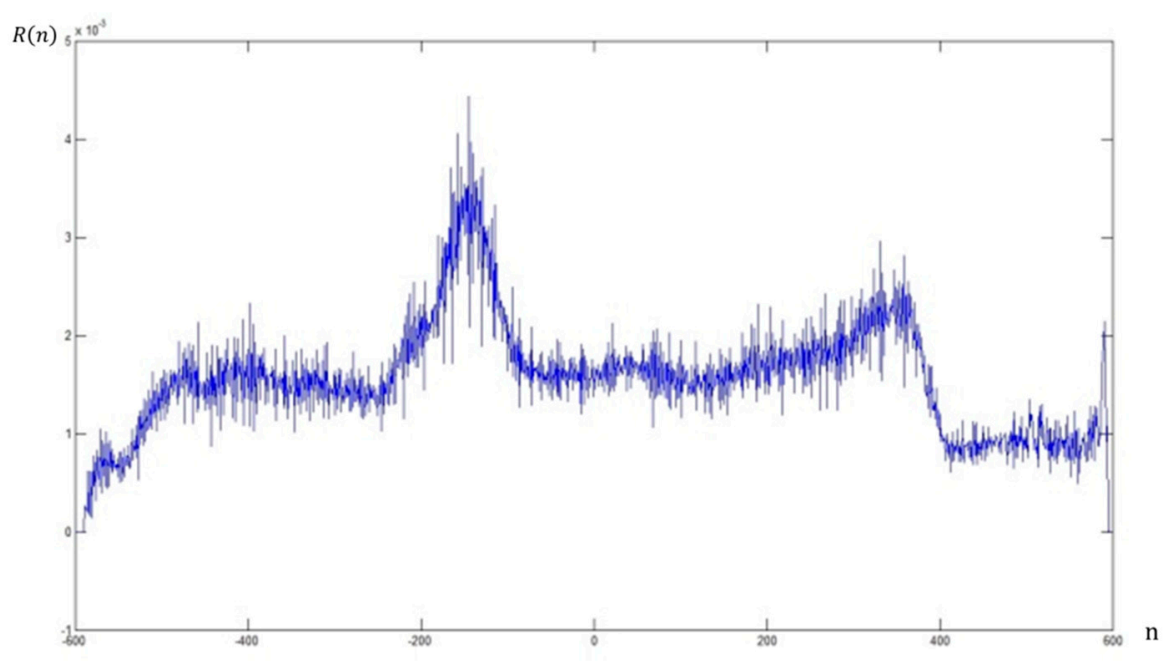

Figure 19. Graph of the cross-correlation coefficient.

Recalling that the solid speed $V \_s$ of $0.532 \mathrm{~m} / \mathrm{s}$ obtained from the simulation using Two-Fluid model, the experimentally measured $V \_e$ of $0.517 \mathrm{~m} / \mathrm{s}$ agrees very well with the CFD simulation, with an error of $2.9 \%$. This can be regarded as a good verification of the CFD results.

\section{Conclusions}

One of the goals of this study is that for an annulus flow in the oil industry we explored systematically the characteristics of the solids flow. Pneumatic conveying in an annular channel is simulated by using Fluent. DPM is adopted to explore the flow of particles with different diameters, as well as in the flow of different air velocities. Simulation results demonstrated that the motion of the small particles is affected much more significantly than large particles at the same air velocity. And large air velocity tends to bring more particles into suspension and flowing in the higher positions in the pipe. This provides an important numerical reference for complicated annular environments in the industry process, such as the cuttings blowing in horizontal oil drilling, which is a main contribution of this paper. 
Another achievement of this study is the mutual verification of the flow characteristics of the solids in the annulus section by both CFD simulations and ECT measurements. The process of sand sliding back in the inclined section is also studied by simulation using Two-Fluid model, and by experimental measurement. The experimentally measured solids distribution and velocity in the inclined section agrees reasonably well with the simulation, which effectively verified the numerical simulation results. This can monitor the speed and concentration of the cuttings in the critical position of the inclined pipe in real time, which can detect the debris blockage in time and raise an alarm.

However, there are still some problems in the above studies, such as the parameter deviation between the simulation set and the practical environment, as well as an extremely weak capacitance signal in experiments. In further studies, the more complete parameters should be considered in simulations and experiments to suit more realistic industry environment.

Author Contributions: Conceptualization, S.L. and W.Z.; Methodology, Y.J.; Software, W.Z.; Validation, T.L., and Q.Z.; Formal Analysis, W.Z. and Y.J.; Investigation, Y.J.; Resources, S.L.; Data Curation, W.Z. and Y.J.; Writing-Original Draft Preparation, Y.J. and W.Z.; Writing-Review \& Editing, S.L.; Visualization, Z.L.; Supervision, S.L.; Project Administration, S.L.; Funding Acquisition, S.L.

Funding: This research was funded by [National Science Fund of China (NSFC)] grant number [61571189] and [Overseas Expertise Introduction Program for Disciplines Innovation in Universities] grant number [B13009]. Wanting Zhou was supported by China Scholarship Council.

Conflicts of Interest: The authors declare no conflict of interest.

\section{References}

1. Gargano, F.; Sammartino, M.; Sciacca, V.; Cassel, K.W. Analysis of complex singularities in high-Reynolds-number Navier-Stokes solutions. J. Fluid Mech. 2014, 747, 381-421. [CrossRef]

2. Obabko, A.V.; Cassel, K.W. Navier-Stokes solutions of unsteady separation induced by a vortex. J. Fluid Mech. 2002, 465, 99-130. [CrossRef]

3. Clercx, H.J.H.; Van Heijst, G.J.F. Dissipation of coherent structures in confined two-dimensional turbulence. Phys. Fluids 2017, 29, 21-43. [CrossRef]

4. Chen, X.P.; Zou, D.Y.; Li, D.J.; Tang, J.; Wei, Y.H.; Wang, W. Study on Downhole Cuttings Adhesion of PDC Bit Based on DPM Model. Chin. Petrol. Mach. 2015, 43, 5-10. (In Chinese)

5. Huang, Y.; Wang, X.D.; Wang, J.Y.; Zhang, R. CFD-based Research on the Local Erosion and Abrasion of Cutting-conveying Pipeline Elbow. Chin. Petrol. Mach. 2014, 42, 6-9. (In Chinese)

6. Zhu, X.H.; Ling, Y.M.; Tong, H. Numerical simulation of DTH bit's gas-solid two-phase flow in air drilling. J. Cent. S. Univ. (Sci. Technol.) 2011, 42, 3040-3047.

7. Zhang, Q.C.; Qing, Q.X.; Liu, J. The cylindrical teeth's topology optimisation design of reverse circulation DTH bit based on gas-solid two-phase flow. Chin. J. Eng. Des. 2014, 21, 340-347. (In Chinese) [CrossRef]

8. Sedrez, T.A.; Decker, R.K.; Silva, M.K.D.; Noriler, D.; Meier, H.F. Experiments and CFD-based erosion modeling for gas-solids flow in cyclones. Powder Technol. 2017, 311, 120-131. [CrossRef]

9. Rossbach, V.; Utzig, J.; Decker, R.K.; Noriler, D.; Meier, H.F. Numerical gas-solid flow analysis of ring-baffled risers. Powder Technol. 2016, 297, 320-329. [CrossRef]

10. Kozolub, P.; Klimanek, A.; Bialecki, R.A.; Adamczyk, W.P. Numerical simulation of a dense solid particle flow inside a cyclone separator using the hybrid Euler-Lagrange approach. Particuology 2017, 31, 170-180. [CrossRef]

11. Deendarlianto; Andrianto, M.; Widyaparaga, A.; Dinaryanto, O.; Khasani; Indarto. CFD Studies on the gas-liquid plug two-phase flow in a horizontal pipe. J. Pet. Sci. Eng. 2016, 147, 779-787. [CrossRef]

12. Zhi, S.; Jing, L.; Li, H. CFD of dilute gas-solid two-phase flow using Lagrangian algebraic slip mixture model. Powder Technol. 2014, 266, 120-128.

13. Jafari, M.; Mansoori, Z.; Avval, M.S.; Ahmadi, G.; Ebadi, A. Modeling and numerical investigation of erosion rate for turbulent two-phase gas-solid flow in horizontal pipes. Powder Technol. 2014, 267, 362-370. [CrossRef]

14. Setyawan, A.; Indarto; Deendarlianto. Experimental investigations of the circumferential liquid film distribution of air-water annular two-phase flow in a horizontal pipe. Exp. Therm. Fluid Sci. 2017, 85, 95-118. [CrossRef] 
15. Xie, Z.; Hewitt, G.F.; Pavlidis, D.; Salinas, P.; Pain, C.C.; Matar, O.K. Numerical study of three-dimensional droplet impact on a flowing liquid film in annular two-phase flow. Chem. Eng. Sci. 2017, 166, 303-312. [CrossRef]

16. Li, Z.X.; Wang, G.Y.; Yousaf, M.; Yang, X.H.; Ishii, M. Flow structure and flow regime transitions of downward two-phase flow in large diameter pipes. Int. J. Heat Mass Transf. 2018, 118, 812-822. [CrossRef]

17. Mccaslin, J.O.; Desjardins, O. Numerical investigation of gravitational effects in horizontal annular liquid-gas flow. Int. J. Multiph. Flow 2014, 67, 88-105. [CrossRef]

18. Aliyu, A.M.; Baba, Y.D.; Lao, L.; Yeung, H.; Kim, K.C. Interfacial friction in upward annular gas-liquid two-phase flow in pipes. Exp. Therm. Fluid Sci. 2017, 84, 90-109. [CrossRef]

19. Setyawan, A.; Indarto; Deendarlianto. The effect of the fluid properties on the wave velocity and wave frequency of gas-liquid annular two-phase flow in a horizontal pipe. Exp. Therm. Fluid Sci. 2016, 71, $25-41$. [CrossRef]

20. Morsi, S.A.; Alexander, A.J. An investigation of particle trajectories in two-phase flow systems. J. Fluid Mech. 2006, 55, 193-208. [CrossRef]

21. Gosman, A.D.; Loannides, E. Aspects of Computer Simulation of Liquid-Fueled Combustors. J. Energy 1983, 7, 482-490. [CrossRef]

22. Yuan, Z.L. Gas Solid Two Phase Flow and Numerical Simulation; Southeast University Press: Nanjing, China, 2013. (In Chinese)

23. Wu, C.W.; Ma, G.J.; Zhou, P. A Review of the Study on the Boundary Slip Problems of Fluid Flow. Adv. Mech. 2008, 38, 265-282. (In Chinese)

(C) 2018 by the authors. Licensee MDPI, Basel, Switzerland. This article is an open access article distributed under the terms and conditions of the Creative Commons Attribution (CC BY) license (http:/ / creativecommons.org/licenses/by/4.0/). 